\title{
ESTUDIODE LAS ZOONOSIS PARASITARIAS DE LOCALIZACIÓN OCULAR EN EL INSTITUTO DE OFTALMOLOGÍA (INO) (PERIODO 1985-1999)
}

\author{
Marco García S. ${ }^{1}$, Amanda Chávez V. ${ }^{2}$, Eva Casas A. ${ }^{2}$, Diego Díaz C. ${ }^{3}$, José \\ Avendaño C. ${ }^{4}$, Betty Campos D. ${ }^{5}$ y Francisco Loayza V ${ }^{6}$
}

\section{A BSTRACT}

The objective of the present study was to determine the frequency of parasitic zoonosis with ocular localization, diagnosed in the Institute of Ophthalmology (INO), which belongs to the Ministry of Health (MINSA) in Lima-Peru, between 1985 to 1999. The analysis of 4,843 histopathology records submitted to the Laboratory of Ocular Pathology between the years 1985 and 1999 , showed that $0.47 \pm 0.002 \%(23 / 4,843)$ corresponded to parasitic zoonosis; where the suggestive lesions of leishmaniosis, toxocariosis and ocular cysticercosis had a frequency of $34.8 \pm 0.19 \%, 30.4 \pm 0.19 \%$ and $17.4 \pm 0.15 \%$, respectively. While the analysis carried out with 1,306 clinical records of patients who went to the Uvea Service of the same institute between the years 1988 and 1999 showed that $22.3 \pm 0.02 \%(291 / 1,306)$ suffered from some type of parasitic zoonosis being the most frequent the uveitis due to toxoplasma $(88.7 \pm 0.04 \%)$, toxocariosis and the ocular cysticercosis $(10.0 \pm 0.03 \%$ and $1.4 \pm 0.01 \%)$. It was concluded that the histopatologic diagnosis showed a low frequency $(0.47 \pm 0.002 \%)$ of parasitic zoonosis; however, a high frequency exists in uveitis patients that attend the INO clinic $(22.3 \pm 0.02 \%)$.

Key words: parasitic zoonosis, uveitis, Institute of Ophthalmology, Public Health, ocular toxoplasmosis

\section{Restimen}

El objetivo del presente estudio fue determinar la frecuencia de las zoonosis parasitarias con localización ocular diagnosticadas en el Instituto de Oftalmología (INO) perteneciente al Ministerio de Salud (MINSA) en Lima - Perú, en el periodo comprendido entre 1985 a 1999. En la primera parte del estudio se evaluaron los registros de 4,843 muestras histopatológicas, remitidas al Laboratorio de Patología Ocular entre los años 1985 y 1999 , encontrándose que un $0.47 \pm 0.002 \%(23 / 4,843)$ correspondieron a zoonosis parasitarias; donde las lesiones sugerentes de leishmaniosis, toxocariosis y cisticercosis oculares tuvieron una frecuencia de $34.8 \pm 0.19 \%, 30.4 \pm 0.19 \%$ y $17.4 \pm 0.15 \%$ respectivamente. En la segunda parte del estudio, se evaluaron las fichas clínicas de 1,306 pacientes que acudieron al Servicio de Uvea del mismo instituto entre los años 1988 y 1999

\footnotetext{
'Práctica privada

${ }^{2}$ Laboratorio de Microbiología y Parasitología, FMV-UNMSM. E-mail: a_chavez_g@hotmail.com

${ }^{3}$ Clínica de Animales Menores, FMV-UNMSM

${ }^{4}$ Laboratorio de Patología Ocular, Instituto de Oftalmología (INO)

'Servicio de Uvea, Instituto de Oftalmología (INO)

${ }^{6}$ Laboratorio de Anatomía Ocular, Instituto de Oftalmología (INO)
} 
encontrándose que un $22.3 \pm 0.02 \%(291 / 1,306)$ padecía algún tipo de zoonosis parasitaria, siendo la más frecuente las uveítis por toxoplasma $(88.7 \pm 0.04 \%)$, además de casos de uveítis por toxocariosis y las cisticercosis oculares $(10.0 \pm 0.03 \%$ y $1.4 \pm 0.01 \%)$. Se puede concluir que el diagnóstico histopatológico muestra una frecuencia baja $(0.47 \%)$ de zoonosis parasitarias, sin embargo, existe una alta frecuencia en los pacientes con uveitis que acuden al consultorio del INO (22.3\%).

Palabras clave: zoonosis parasitarias, uveítis, Instituto de Oftalmología, Salud Pública, toxoplasmosis ocular

\section{INTRODUCCIÓN}

Tanto en el Perú como en otros países de América Latina, los problemas sanitarios constituyen una de las grandes brechas en el desarrollo de la sociedad. El aumento en la frecuencia de enfermedades zoonóticas de tipo parasitario en nuestro medio tiene mucha importancia en Salud Pública. Enfermedades como la toxoplasmosis y la toxocariasis humana presentan una alta morbilidad, fuera de un gran número de casos subclínicos que pasan encubiertos por largo tiempo. Por otro lado, se encuentra la cisticercosis humana, enfermedad de alta frecuencia en el país. La transmisión al hombre de las enfermedades antes mencionadas se da mayormente por contacto directo con animales domésticos o silvestres portadores de los agentes infecciosos, así como en forma indirecta por consumode alimentos contaminados y mal cocidos (cisticercosis porcina).

Los problemas oculares constituían un $10 \%$ de los ingresos hospitalarios en el Perú en 1996. Las zoonosis parasitarias como toxocariasis y toxoplasmosis son consideradas a nivel mundial como causas importantes de problemas oculares (cuadros clínicos de disminución de la visión y de ceguera). Estudios realizados en diversos países, relacionados con las causas de problemas oculares, indican que la toxoplasmosis es una de las principales causas infecciosas de uveítis (lesión que conlleva a serios problemas de visión). Otras enfermedades como la toxocariosis ocular que afecta principalmente a niños y adolescentes, ocasiona un cua- dro clínico similar a una enfermedad tumoral llamada retinoblastoma, que sin tratamiento inmediato es de curso mortal.

El presente estudio tuvo como objetivo determinar la frecuencia de zoonosis parasitarias con localización ocular, diagnosticadas en el Instituto de Oftalmología (INO) perteneciente al Ministerio de Salud (MINSA), durante el periodo comprendido entre enero de 1985 a diciembre de 1999. Para este fin se realizó un estudio retrospectivo los archivos clínicos e histopatológicos del Instituto.

\section{Materiales y Métodos}

El estudio se realizó en base a fichas clínicas del Laboratorio de Patología Ocular "Michael Hogan" y del Servicio de Uvea, ambos del Instituto de Oftalmología (INO) del Ministerio de Salud, Lima.

\section{Laboratorio de Patología Ocular}

Se realizó un estudio retrospectivo con 4,843 protocolos de histopatología. Estas muestras fueron recepcionadas durante el periodo de enero de 1985 a diciembre de 1999. Cada uno de estos protocolos incluyó en forma adicional la ficha clínica (historia clínica, protocolos de uveítis y antecedentes personales), pruebas diagnósticas (ecografía ocular, serología y fondo de ojo) y análisis de laboratorio (hemogramas), tomando como referente diagnóstico el criterio del médico oftalmólogo a cargo. 
Cuadro 1. Frecuencia de zoonosis parasitarias oculares encontradas en el Laboratorio de Patología Ocular del Instituto de Oftalmología (INO) durante el periodo 1985 1999 ( $n=4,843$ protocolos)

\begin{tabular}{lccccc}
\hline \multirow{2}{*}{ Zoonosis parasitaria } & \multirow{2}{*}{$\begin{array}{c}\mathrm{N}^{\circ} \mathrm{de} \\
\text { casos }\end{array}$} & \multirow{2}{*}{$\begin{array}{c}\text { Frecuencia } \\
(\%)\end{array}$} & \multicolumn{2}{c}{ Edad (años) } & \multirow{2}{*}{$\begin{array}{c}\text { Género } \\
\text { (\% en }\end{array}$} \\
\cline { 5 - 6 } & 8 & $34.8 \pm 0.19$ & $21-38$ & 28.5 & 100.0 \\
Leishmaniosis & 7 & $30.4 \pm 0.19$ & $5-18$ & 10.8 & 57.1 \\
Larva migrante ocular & 5 & $17.4 \pm 0.15$ & $10-36$ & 22.0 & 20.0 \\
Cisticercosis ocular & 2 & $8.7 \pm 0.12$ & $4-5$ & 4.5 & 50.0 \\
Hidatidosis orbitaria & 2 & $8.7 \pm 0.12$ & & 5 días & 100.0 \\
Toxoplasmosis congénita & & & & & \\
\hline
\end{tabular}

Las variables bajo estudio fueron el diagnóstico histopatológico, edad, género y tipo de lesión, a fin de determinar la frecuencia de casos de zoonosis parasitarias. Se analizaron cuadros de frecuencia por el método de chi cuadrado.

\section{Servicio de Uvea}

Se realizó un estudio retrospectivo con 1,306 protocolos de uveítis. Estos casos correspondieron a pacientes que fueron atendidos en el periodo comprendido entre los años 1988 y 1999. Las fichas clínicas consignan información referida a enfermedades padecidas por el paciente o sus familiares como diabetes y tuberculosis, así como el contacto del paciente con animales, consumo o manejo de cames crudas, y consumo de leche fresca.

Las variables en estudio fueron el diagnóstico clínico, género, edad, procedencia, ocupación, ojo afectado, tipo de lesión, posibles vías de contacto, y sintomatología.

Se aplicó la prueba del chi cuadrado para determinar relaciones significativas entre las variables bajo estudio.

\section{Resultados}

\section{Laboratorio de Patología Ocular}

Se encontró que el $0.47 \pm 0.002 \%$ (23/4,843 muestras) eran compatibles con lesiones concordantes con parasitismo ocular. La distribución de los casos diagnosticados según el tipo de parasitismo, edad y género del paciente se muestra en el Cuadro 1.

Los casos diagnosticados en el Servicio de Uvea del Instituto de Ofalmología en el periodo en estudio se muestran en el Cuadro 2. Se determinó que el $56.6 \pm 0.03 \%$ (739/ $1,306)$ de uveítis se debió a causas infecciosas, el $30.7 \pm 0.03 \%(401 / 1,306)$ por causas no infecciosas y el $13.1 \pm 0.02 \%(171 / 1,306)$ por etiologías inespecíficas. Las uveítis debidas a zoonosis parasitarias comprendieron el $22.3 \%$ del total de causas de uveítis y el $39.4 \%$ de las causas infecciosas.

La distribución de los casos diagnosticados según el tipo de parasitismo, edad y género del paciente se muestra en el Cuadro 3. No se encontró diferencias estadísticas significativas $(\mathrm{P}>0.05)$ por género del paciente. 
Cuadro 2. Distribución según año y etiología de los casos de uveítis por causas infecciosas diagnosticadas por el Servicio de Uvea del Instituto de Oftalmología durante el periodo 1988-1999. Lima-Perú

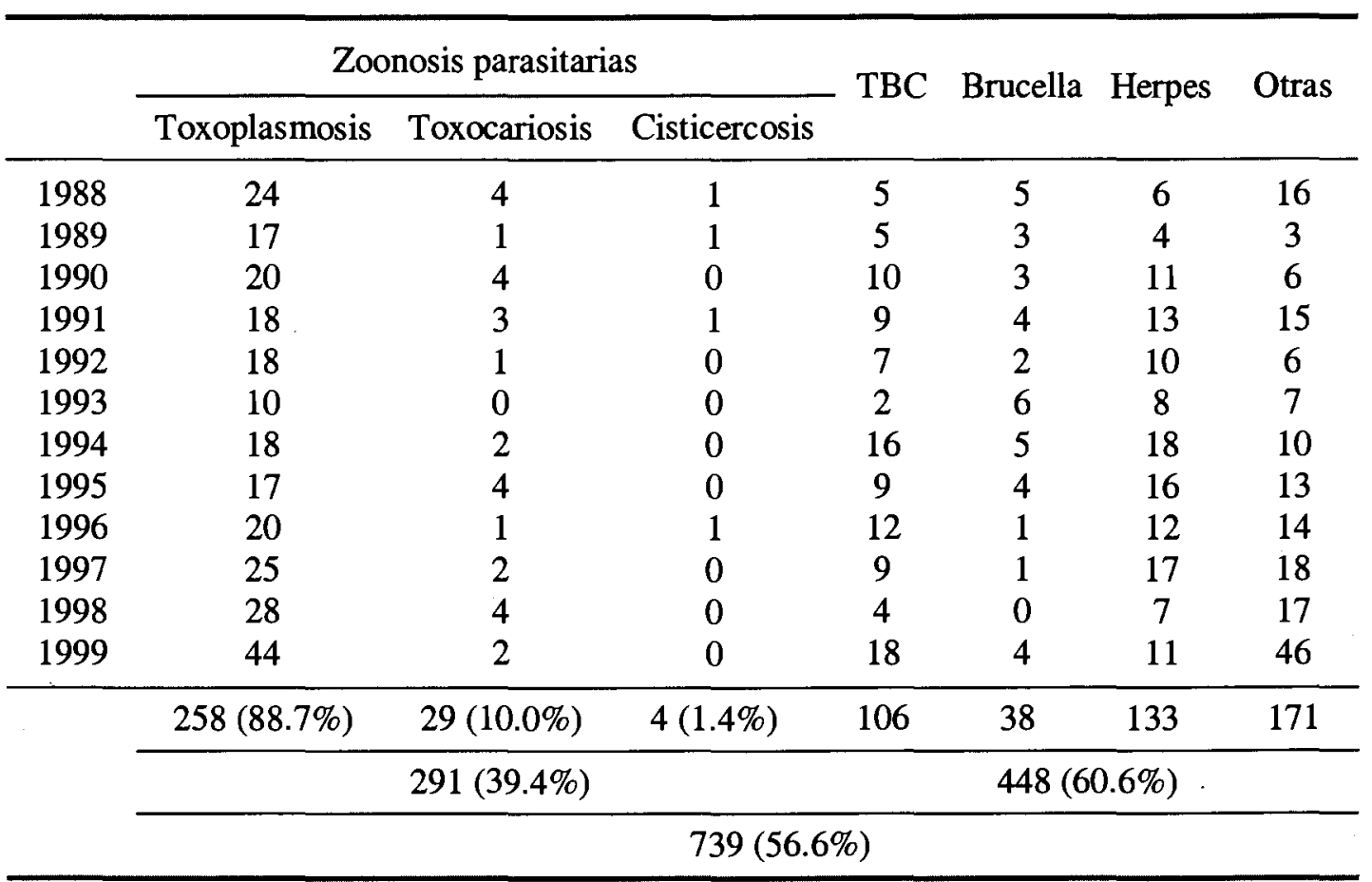

Cuadro 3. Frecuencia de zoonosis parasitarias oculares encontradas en el Servicio de Uvea del Instituto de Oftalmología (INO) durante el periodo 1988-1999 $(n=1,306$ protocolos)

\begin{tabular}{|c|c|c|c|c|c|}
\hline \multirow{2}{*}{ Zoonosis parasitaria } & \multirow{2}{*}{$\begin{array}{l}\mathrm{N}^{\circ} \mathrm{de} \\
\text { casos }\end{array}$} & \multirow{2}{*}{$\begin{array}{c}\text { Frecuencia } \\
(\%)\end{array}$} & \multicolumn{2}{|c|}{ Edad (años) } & \multirow{2}{*}{$\begin{array}{c}\text { Género } \\
\text { ( } \% \text { en } \\
\text { varones) }\end{array}$} \\
\hline & & & Rango & Prom & \\
\hline Toxoplasmosis & 258 & $88.7 \pm 0.04$ & $3-70$ & 30.9 & 49.6 \\
\hline Toxocariosis & 29 & $10.0 \pm 0.03$ & $4-43$ & 13.5 & 55.2 \\
\hline Cisticercosis ocular & 4 & $1.4 \pm 0.01$ & $15-36$ & 26.5 & 100.0 \\
\hline
\end{tabular}

Los casos de toxocariosis y cisticercosis ocular fueron unilaterales $(51.7$ y $100 \%$ en el ojo izquierdo, respectivamente), en tanto que se encontró el $17.0 \%$ de afecciones en ambos ojos en los casos de uveítis toxoplásmicas. No se encontró diferencias estadísticas con relación al ojo afectado. 
Los pacientes con toxoplasmosis eran mayormente de Lima (63.3\%), seguido de Cajamaca, Piura y San Martín. En forma similar, la mayor procedencia de los casos de toxocariosis y cisticercosis fue de Lima (69.0\% y $3 / 4$, respectivamente).

La mayor parte de los pacientes con toxoplasmosis eran estudiantes, ya sea en edad escolar o superior $(31.4 \%$ ) y amas de casa $(22.1 \%)$, en tanto que en el caso de toxocariosis eran estudiantes de educación superior $(48.3 \%)$, escolares (37.9\%) y niños en edad preescolar $(6.9 \%)$.

\section{Discusión}

La aparición de nuevas técnicas diagnósticas para detectar problemas oculares en los últimos 20 años, ha traído la disminución del uso de técnicas como la enucleación, usada como alternativa quirúrgico-terapéutica en afecciones como la retinoblastoma y oftalmia simpática; que luego de una uveítis traumática por injuria perforante en uno de los ojos provoca un cuadro similar de uveítis en el otro ojo. Esto se cree que es debido a una causa autoinmune y que sin tratamiento inmediato conllevan a tisis bulbar y pérdida del ojo (Vaughan et al., 1992).

Debe considerarse que debe hallarse el parásito para realizar un diagnóstico histopatológico definitivo de problemas oculares causados por zoonosis parasitarias. No basta dar un diagnóstico presuntivo por lesiones sugerentes, de allí que para el caso de una toxocariosis ocular deba realizarse un examen seriado de la muestra, aspecto que encarece el examen de laboratorio. Éste es un aspecto que debe tenerse en cuenta cuando se comparan los datos hallados en el presente estudio con aquellos de Doria (1984) en un estudio realizado con anterioridad en el mismo laboratorio.

La principal zoonosis parasitaria encontrada en el Laboratorio de Patología Ocular fue la leishmaniosis (34.8\%). Sin embargo, esto se debió principalmente al traslado al Instituto de Oftalmología en 1991 de ocho pacientes de una zona agropecuaria fronteriza entre Arequipa y Cusco. Si se excluyen estos casos podría considerarse a la toxocariosis como principal causa de problemas oculares causados por zoonosis parasitarias que son diagnosticadas en este laboratorio.

Las causas de las uveítis que pudieron ser determinadas entre los pacientes que acudieron al Servio de Uvea entre enero de 1988 a diciembre de 1999 fueron debidas principalmente a infecciones, ya sean adquiridas o congénitas a $T$. gondii $(19.8 \%)$, sobrepasando a otros problemas infecciosos, traumáticos, autoinmunes y enfermedades sistémicas. Este resultado concuerda con lo descrito en la literatura donde se señala a la toxoplasmosis como una causa importante de problemas oculares (Cordero-Moreno, 1993; Hay y Dutton, 1995); e incluso es considerada la causa más común de retinocoroiditis, constituyendo el $28 \%$ de los casos de uveítis posterior (Vaughan et al., 1992).

La toxoplasmosis ocular se presentó en un amplio rango de edades (3-70 años), y con un promedio de 29.6 años. La mayor cantidad de diagnósticos comprendió a pacientes entre 20 y 40 años. Se asume que la mayoría de los casos de toxoplasmosis oculares son de tipo congénito, debido a que diversos autores concuerdan en que en la forma adquirida la afección más común es de tipo ganglionar (forma linfadenopática febril), y que en la forma congénita se aprecian cuadros severos, que cursan con meningoencefalitis (pacientes inmunocompetentes) y retinitis (Acha y Cifres, 1992; Cordero-Moreno, 1993).

La presencia de casos de uveítis toxoplásmicas fue en su mayoría del departamento de Lima (64.3\%), en especial en los distritos de San Juan de Lurigancho, Cercado y la Victoria. Sin embargo, esta información se encuentra sesgada por el hecho de la cercanía de estos lugares a los servicios 
oftalmológicos, y que en muchas áreas rurales del interior del país no cuentan con dichos servicios.

\section{Conclusiones}

En el Laboratorio de Patología Ocular del Instituto de Oftalmología se encontró una baja frecuencia $(0.47+0.002 \% ; 23 / 4,843)$ de muestras con diagnóstico de lesiones sugerencias de zoonosis parasitarias.

La frecuencia de casos de zoonosis parasitarias en el servicio de Uvea del mismo Instituto se encontraron $22.3 \pm 0.02 \%$ (291/1,306 pacientes). Las uveitis toxoplásmicas fueron las causas más frecuentes de uveitis (19.75\%).

\section{Literatura Crtada}

1. Acha, P.; B. Szyfres. 1992. Zoonosis y enfermedades transmisibles comunes al hombre y a los animales. $2^{\mathrm{a}}$ ed. Organización Panamericana para la Salud. Washington, EE.UU. 989 p.

2. Cajas, J. 1999. Contaminación de los parques públicos del Cono Sur: Chorri llos, San Juan de Miraflores y Villa el Salvador, con huevos de Toxocara spp. Tesis Bach. Fac. Med. Vet., Univ. Nac. Mayor de San Marcos. Lima. 77 p.

3. Cordero-Moreno, R. 1993. Manifestaciones oculares de algunas enfermedades tropicales. Publicaciones de la Se- cretaría de la Universidad Central de Venezuela. Caracas - Venezuela. 294 p.

4. Doria, D.M. 1984. Zoonosis parasitarias con localización ocular en Lima Metropolitana. Tesis Bach. Fac. Med. Vet., Univ. Nac. Mayor de San Marcos. Lima. $27 \mathrm{p}$.

5. Hay, J.; G. Dutton. 1995. Toxoplasma and the eye. Brit. Med. Journal 310: 1021$1022 \mathrm{p}$.

6. La Rosa, V. 2000. Contaminación de los parques públicos del Cono Norte: Ancón, Carabayllo, Comas, Independencia, Los Olivos, Puente Piedra, Rímac, San Martín de Porres y Santa Rosa, con huevos de Toxocara sp. Tesis Bach. Fac. Med. Vet., Univ. Nac. Mayor de San Marcos. Lima. 60 p.

7. Minvielle, M.; G. Niedfeld; M. Ciarmela; J. Basualdo. 1999. Toxocariasis causada por Toxocara canis: aspectos epidemiológicos. Enferm. Infec. Microbiol. Clin. 17: 300-306.

8. Serrano, M. 1999. Contaminación de los parques públicos del Cono Este: Ate, Chaclacayo, Cieneguilla, El Agustino, La Molina, San Juan de Lurigancho y Santa Anita con huevos de Toxocara sp. Tesis Bach. Fac. Med. Vet., Univ. Nac. Mayor de San Marcos. Lima. 73 p.

9. Vaughan, D.; T. Asbury; P. RiordanEva. 1992. Oftalmología general. Ed. Manual Moderno. México. $530 \mathrm{p}$.

10. Velarde, J. 1999. Contaminación de los parques públicos de la provincia Constitucional del Callao con huevos de Toxocara $s p$. Tesis Bach. Fac.Med. Vet., Univ. Nac. Mayor de San Marcos. Lima. 62 p. 